\title{
LUT
}

University

\section{Performance of Multi-carrier Technology over VHF Channels for Rural Area Applications}

Carrillo Melgarejo Dick, Fraidenraich Gustavo, Quirino Luiz, Medeiros Alvaro, Nardelli Pedro

This is a Final draft

version of a publication

published by IEEE

in 2019 IEEE Wireless Communications and Networking Conference Workshop (WCNCW)

DOI: $10.1109 /$ WCNCW.2019.8902782

Copyright of the original publication: (C) IEEE 2019

Please cite the publication as follows:

Carrillo Melgarejo, D., Fraidenraich, G., Quirino, L., Medeiros, A., Nardelli, P. (2019).

Performance of Multi-carrier Technology over VHF Channels for Rural Area Applications. In: 2019 IEEE Wireless Communications and Networking Conference Workshop (WCNCW), Marrakech, Morocco, 2019. DOI: 10.1109/WCNCW.2019.8902782

(C) 2019 IEEE. Personal use of this material is permitted. Permission from IEEE must be obtained for all other uses.

This is a parallel published version of an original publication. This version can differ from the original published article. 


\title{
Performance of Multi-carrier Technology over VHF Channels for Rural Area Applications
}

\author{
Dick Carrillo Melgarejo $^{1}$, Gustavo Fraidenraich ${ }^{2}$, Luiz Quirino ${ }^{3}$, Alvaro Medeiros ${ }^{3}$, Pedro Nardelli ${ }^{1}$ \\ ${ }^{1}$ School of Energy Systems, LUT University, Finland \\ ${ }^{2}$ School of Electrical and Computer Engineering, University of Campinas, Brazil \\ ${ }^{3}$ Federal University of Juiz de Fora, Brazil
}

\begin{abstract}
This paper focuses on the application of multicarrier technologies in rural area communications on very high frequency (VHF) channels. Our target is to analyze the performance of typical long-term evolution (LTE) technology on three specific rural scenarios: (i) high density of users in specific regions, (ii) high inter-cell interference regions, and (iii) high demand of similar services. These scenarios operate in very high frequency (VHF) channels to support rural connectivity.

Numerical results are obtained via system-level simulations using the ns-3 simulator, whose settings were calibrated based on real LTE deployments on VHF.

We specifically assess the performance of available frequency reuse techniques showing that typical solutions for cellular networks may lead to remarkable throughput differences in these extreme scenarios. The simulation results showed that hard frequency reuse - which has a poor performance in typical scenarios - outperforms all other studied options in the proposed study-case.
\end{abstract}

\section{INTRODUCTION}

Multi-carrier communication techniques are nowadays employed in fourth generation of mobile network (4G) systems that are based on orthogonal frequency-division multiple access (OFDMA) [1], and shall be part of the future fifth generation of mobile network (5G) [2]. Due to its popularity, OFDMA becomes an attractive solution for rural communication in many regions of large developing countries (e.g. Brazil, Russia, India, China) and also other sectors of Northern Europe [3]. Commercial cellular network solutions that today operate in ultra high frequency (UHF) are also being deployed in very high frequency (VHF) to achieve coverage cells with radios up to $50 \mathrm{~km}$ [4], [5]. This scenario opened great opportunities to create multi-carrier network coverage in rural areas that are used to support specific internet of things (IoT) applications [6]-[8].

Brazil is an instructive example in applying multi-carrier technologies over VHF in rural areas [4], where cellular networks are deployed by agribusiness companies with the right to operate in specific VHF channels. This right is conditioned to the fact that connectivity could not be further commercialized to any third-party [9]. It means that to increase the return on investment (ROI), it is advisable to have an accurate analysis of network performance, specially in some specific scenarios; they are usually IoT devices that are installed in specific regions of interest and in industrial machines that take part of industrial production processes [10], [11].
Although such networks are already in use in few cases, there is still a lack of research that focuses on the performance of multi-carrier techniques in such rural scenarios. This paper tries to fill this gap, specially now that IoT applications are becoming increasingly popular in rural areas to be applied in vertical scenarios as agribusiness and electric energy distribution. In specific terms, we showed via system-level simulation (calibrated with real network measurements) that there is big room for improvement in the network performance (evaluated in terms of aggregate throughput) by comparing popular techniques of frequency reuse to reduce self-interference. Our numerical results evince the existing opportunities to finetune the system parameters in "extreme" cases related to rural applications.

The rest of this paper is divided as follows. The definition of the extreme scenarios is presented in Section II. Section III describes the system model while Section IV presents the simulation methodology and the particular study case of frequency reuse. Section V contains the numerical results. The conclusions and future works are described in Section VI.

\section{EXTREME SCENARIOS AND MOTIVATION}

\section{A. Extreme Scenarios}

Let us consider a multi-carrier cellular network deployed in VHF that aims to enable rural connectivity, specifically IoT applications in energy systems. Several electric energy distribution scenarios are typically concentrated in specific regions by specific periods of time. This means that users load are not uniformly distributed in all coverage areas, as it is expected in typical cellular networks. Other important fact is that, depending on the IoT application, the mobile users are devices with deterministic behavior (i.e. sending short messages in specific times) [12], which is different in the traditional human-to-human communication. The combination of all these particular characteristics, described before, generates some extreme scenarios that deserve special attention.

High users density in specific regions: In the particular case of substations that are part of electric power distribution systems where most of the devices are distributed typically in mosaic form (Ex. rectangular form). Inside this form, most of the cellular users are concentrated, creating an extreme scenario, untypical in commercial UHF cellular networks. This extreme scenario in some cases could represent most of the $90 \%$ of the network load, creating an unbalanced user density. 
Fig. 1(a) exemplifies this scenario, emphasizing the fact that the networks operates in VHF.

High dense inter-cell interference regions: Other extreme scenario is characterized by the fact that in some cases the region of interest is located in the frontier of two different cells, which implies a low signal-to-interference-and-noise ratio (SINR) region. The particularity of this scenario is that cellular users will almost always be inside the geometry region of interest (typical a square), generating a region of high interference. Fig. 1(b) shows this scenario.

Users demanding very similar services: Many IoT applications that use multi-carrier technologies demand today high throughput data type as in video streaming. This applicationrelated demand may be (and usually is) correlated across users: many machines use the same application at the same time. This means that scheduler algorithms and quality of service (QoS) policies should deal with this particular kind of congestion, which are related to specific region of interested characterized by a high user density. Fig. 1(c) presents an example of many users demanding video traffic into a geometric arbitrary region.

It is important to emphasize that these scenarios are strictly correlated and define the "extreme scenarios" to be studied in the paper. As to be discussed later on, these scenarios will be studied using the Network simulator 3 (ns-3), which is an accurate system-level simulator [13] that considers the commercial Long-Term Evolution (LTE) protocol stack.

\section{B. Motivation}

In contrast of what is studied in commercial UHF cellular networks where users are deployed uniformly in all coverage network (Fig. 5). We would like to take the attention of these extreme scenarios (in VHF) where users distribution is always limited by a specific geometry form (in this case a rectangle) inside the expected cellular network coverage(Fig. 6).

Therefore, some metrics are generated in order to show that specific network optimization could be applied to increase network performance (for example throughput). This optimization results generated by simulation indicate that there is room for potential scientific analyze on these extreme scenarios.

\section{SySTEM MODEL}

The networks deployed in VHF are based on the protocol stack of LTE, that is why that the system model is based on the system architecture evolution (SAE) [14]. In this section we focused on providing important details of the algorithm used to calculate the SINR. Our system model is based on LTE/SAE architecture. This architecture contains two main evolved subsystems: evolved UTRAN (E-UTRAN) and evolved packet core (EPC) [15]. Both are described in Fig. 2 and they are summarized in following lines.

EPC: It is also know as core network. The EPC also can be connected to other 3rd Generation Partnership Project (3GPP) and non-3GPP radio-access networks. The EPC consists of one control-plane node, called mobility management entity (MME), and two user-plane nodes, called serving gateway (S-GW) and packet-data network gateway (P-GW) [16].

\section{Base Station}

operating in VHF
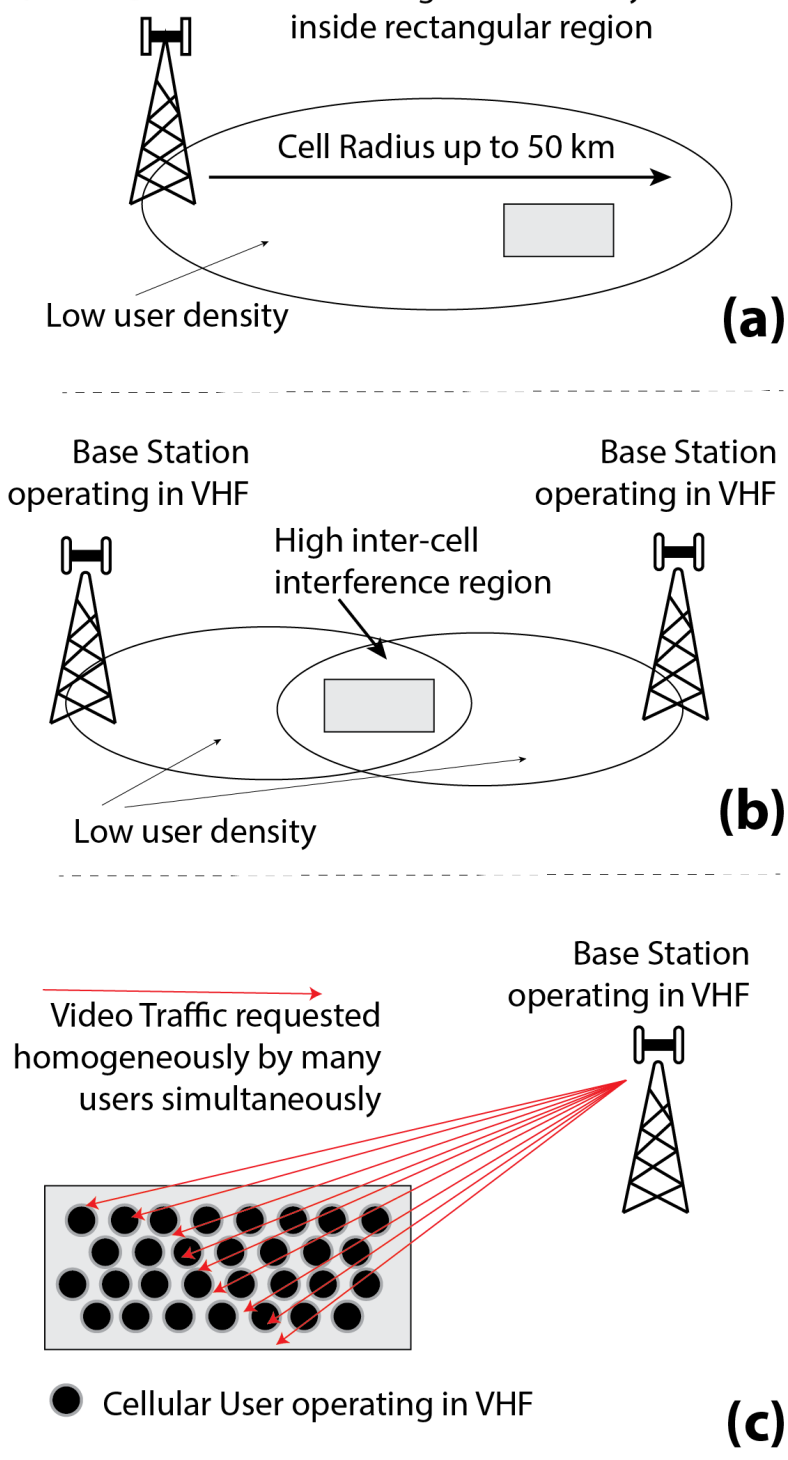

Fig. 1. Description of rare scenarios in agribusiness market. (a) High density of users in specific regions (mosaic). (b) High inter-cell interference regions. (c) Users demanding very similar services.

E-UTRAN: The E-UTRAN consists of the base stations, denoted as evolved NodeB (eNodeB), that are connected to each other through the X2 interface and to the EPC through the $\mathrm{S} 1$ interface [17].

The software ns-3 is used to simulate SAE architecture [13]. This simulation software allows deployments of almost realtime scenarios based on discrete events. The simulator includes an error model for physical downlink shared channel (PDSCH) based on the link-to-system mapping (LSM) technique [18]. LSM is based on the mapping of single link layer performance obtained with link level simulators. For this purpose the SINR is used as an input of a function that depends of transport block (TB) length, modulation code scheme (MCS), effective code rate $(\mathrm{ECR})$ that represents the actual transmission rate 


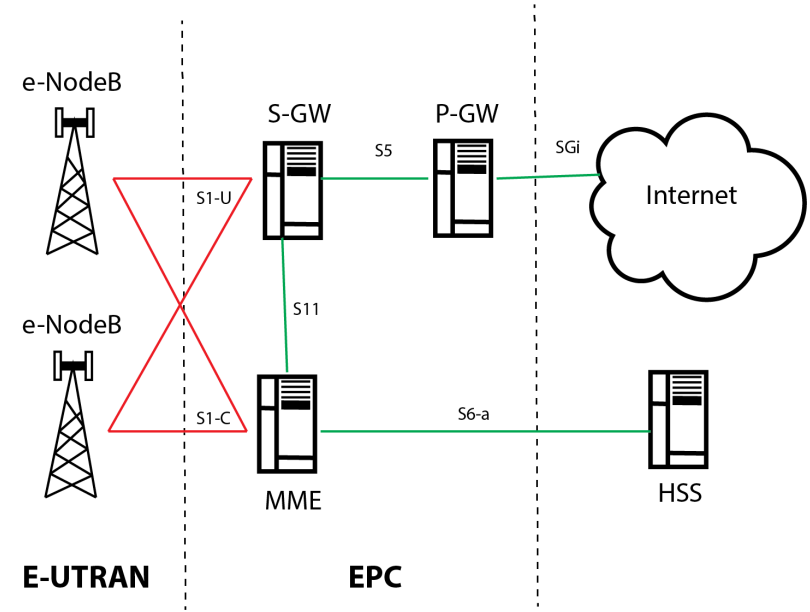

Fig. 2. LTE Architecture based on SAE. It is composed by two main elements: E-UTRAN and EPC.

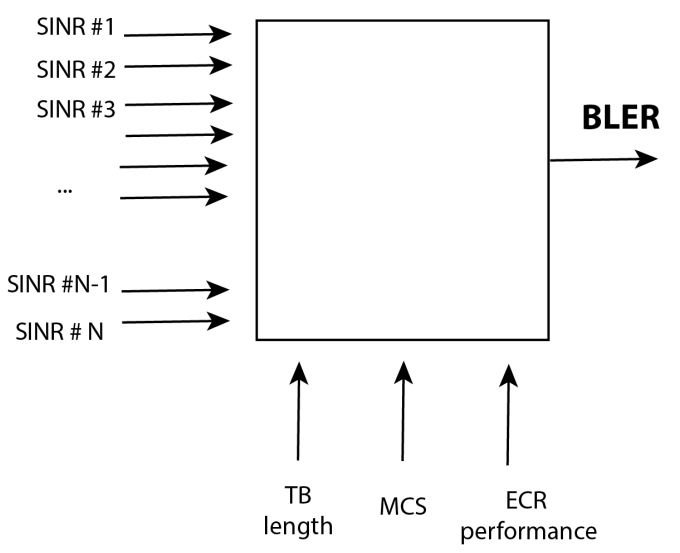

Fig. 3. Physical error model considered in ns-3.

according to the channel coding and MCS. The output of this function is the block error rate (BLER), which is going to be used to calculate users throughput. A block diagram of this error model is described in Fig. 3.

The physical error model is based on the SINR. The SINR of $m$ th target user $(m=1,2, \cdots, N)$ at the $k$ th sub-carrier is calculated as

$$
\operatorname{SINR}^{m}(k)=\frac{P_{\mathrm{RX}}^{m}(k)}{\sigma_{n}^{2}+P_{\mathrm{CCI}}^{m}(k)},
$$

where $\sigma_{n}^{2}$ is the per subcarrier AWGN power level, $P_{\mathrm{CCI}}^{m}(k)$ is the total co-channel interference (CCI) power level and $P_{\mathrm{RX}}^{m}(k)$ is the received signal power level, both at the $k$ th subcarrier for $m$ th target user.

The two latest variables are defined in eq. (3) and eq. (2), as follows. The total CCI power level $P_{\mathrm{CCI}}^{m}(k)$ at the $k$ th subcarrier for $m$ th target user is

$$
P_{\mathrm{RX}}^{m}(k)=\frac{P_{\mathrm{TX}}^{m} \cdot P_{\text {loss }}^{m} \cdot\left|h_{m}(k)\right|^{2}}{N_{\text {load }}(j)},
$$

where $N_{\text {load }}(j)$ is the total number of co-channel subcarriers for the $j$ th OFDMA symbol. $P_{\text {loss }}^{m}$ is the path loss including shadowing and antennas gains, $P_{\mathrm{TX}}^{m}$ is the total transmit power from eNodeB.

The received signal power level $P_{\mathrm{CCI}}^{m}(k)$ at the $k$ th subcarrier for $m$ th target user is

$$
P_{\mathrm{CCI}}^{m}(k)=\sum_{l=2}^{N_{\mathrm{CCI}}} P_{\mathrm{CCI}}^{l, m}(k)=\sum_{l=2}^{N_{\mathrm{CCI}}} \frac{\left|h_{l, m}(k)\right|^{2} \cdot P_{\text {loss }}^{l, m} P_{\mathrm{TX}}^{l}}{N_{\text {load }}(j)},
$$

where $P_{\mathrm{CCI}}^{l, m}(k)$ is CCI power level from the $l$ th interferer to $m$ th target user, and $N_{\mathrm{CCI}}$ is a number of co-channel interferers and $N_{\text {CCI }} \leqslant N-1$. Details of MCS, SINR, TB length, ECR are out of the scope of this work, however, specific information can be found in [19].

\section{Simulation methodology}

\section{A. Methodology to simulate a semi-real LTE scenario in VHF}

1) Empirical data and system simulator calibration: Realworld performance metrics were collected through drive tests in order to calibrate the system level simulator. The data were obtained in a rural area in São Paulo, Brazil, in 2017/18 as part of a project that considers LTE protocol stack in VHF channels [4]. In this particular case, we used the SINR and throughput to set up the path-loss parameters in ns-3 software. The real world measurements were done in key geographical points located in the edge cell or in points near the eNodeB. Other important measurements were done in high interference regions. All these points were mapped in the system level simulator in order to calibrate the path-loss model. In this particular case, we simplified the calibration using the popular Friis propagation loss model defined by:

$$
P_{r}=\frac{P_{t} G_{t} G_{r} \lambda^{2}}{(4 \pi d)^{2} L},
$$

where $P_{r}$ is the reception power in Watts, $P_{t}$ is the transmission power in Watts, $G_{t}$ is unit-less transmission gain, $G_{r}$ is the unit-less reception gain, $\lambda$ is the wavelength in meters, $d$ is the distance between the eNodeB and the user in meters, and finally the unit-less $L$ is the system loss. The system loss $(L)$ is the parameter that was used to calibrate the system simulator using real world measurements.

TABLE I

PARAMETERS USED IN THE SYSTEM LEVEL SIMULATION

\begin{tabular}{|l|l|}
\hline Parameter name & Value \\
\hline \hline Simulation Time & 5 seconds \\
\hline System Bandwidth & $5 \mathrm{MHz}$ \\
\hline Frequency Operation & $250 \mathrm{MHz}$ (VHF) \\
\hline eNodeB - Tx Power & $43 \mathrm{dBm}$ \\
\hline UE - Tx Power & $23 \mathrm{dBm}$ \\
\hline eNodeB - Noise Figure & $5 \mathrm{~dB}$ \\
\hline UE - Noise Figure & $9 \mathrm{~dB}$ \\
\hline Number of Users & between 3 until 150 Users \\
\hline Data Rate per User (DL) & 1 mega bits per second (Mbps) \\
\hline
\end{tabular}




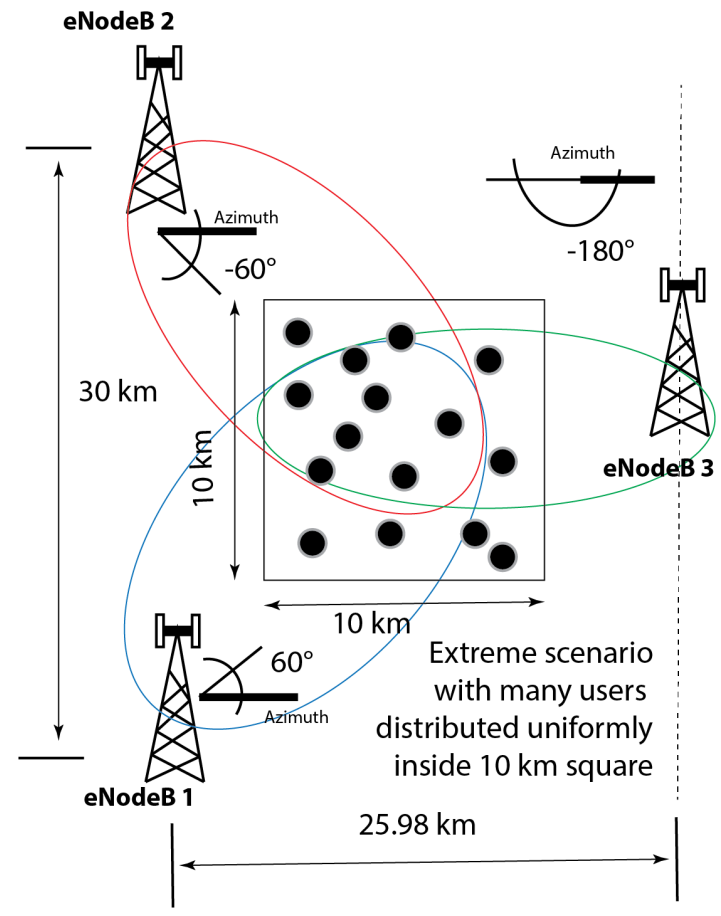

Fig. 4. Simulation setup composed by three eNodeBs with different azimuth values that force a region with high inter-cell interference, over this region is defined a square region which will be populated with different number of users using a random uniform model.

2) Simulation scenario and performance metric: To evaluate the LTE protocol stack operating in VHF for extreme scenarios, we considered the simulation setup described in Fig. 4. We considered three eNodeBs, each one is operating with just one sector, which are using arbitrary azimuth and distances values with the intention to generate a high inter-cell interference region. We assume a high inter-cell interference region as a square of $10 \mathrm{~km}$ each side. Inside this square, cellular users are uniformly distributed. The first eNodeB antenna is using an azimuth of $60^{\circ}$, the second considers a $-60^{\circ}$ of azimuth, and finally the $3 \mathrm{rd}$ eNodeB considers an azimuth of $-180^{\circ}$.

Table I lists the relevant system parameters used in the simulations. These values are calibrated with real-world setups. A downlink transmission (i.e. eNodeB is the transmitter) where every user receives a data rate of $1 \mathrm{Mbps}$ is simulated with the number of simultaneous users that varies between 3 until 150 .

\section{B. Showcase Analysis for Rare Scenarios in VHF}

We analyze the extreme scenarios previously discussed looking at fractional frequency reuse techniques. These techniques are used to manage inter-cell interference in commercial multi-carrier networks, and we will use the same configuration setup to compare throughput performance of typical (commercial) and extreme scenarios. The frequency reuse techniques considered in this study are [20]:

- Hard Frequency Reuse,
- Strict Frequency Reuse,

- Soft Frequency Reuse,

- Soft Fractional Frequency Reuse,

- Enhanced Fractional Frequency Reuse.

1) Study-case 1: The goal is to compare a typical multicarrier cellular scenario (Fig. 5) with an extreme scenario (Fig. 6). For both cases, it is obtained the total aggregated throughput. This case is also used to indicate that these extreme scenarios deserve special attention.

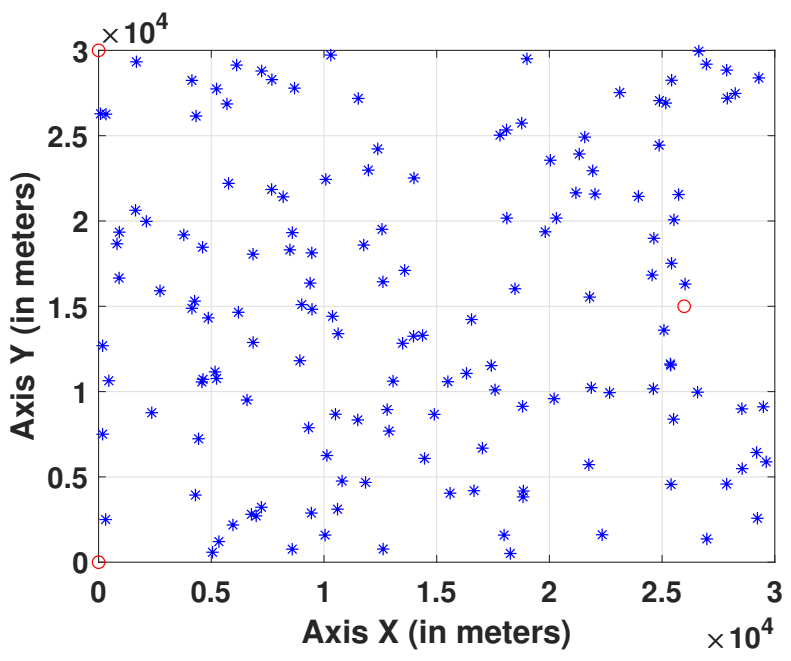

Fig. 5. A typical cellular network scenario. The blue points represent the users. The red points represent the eNodeBs.

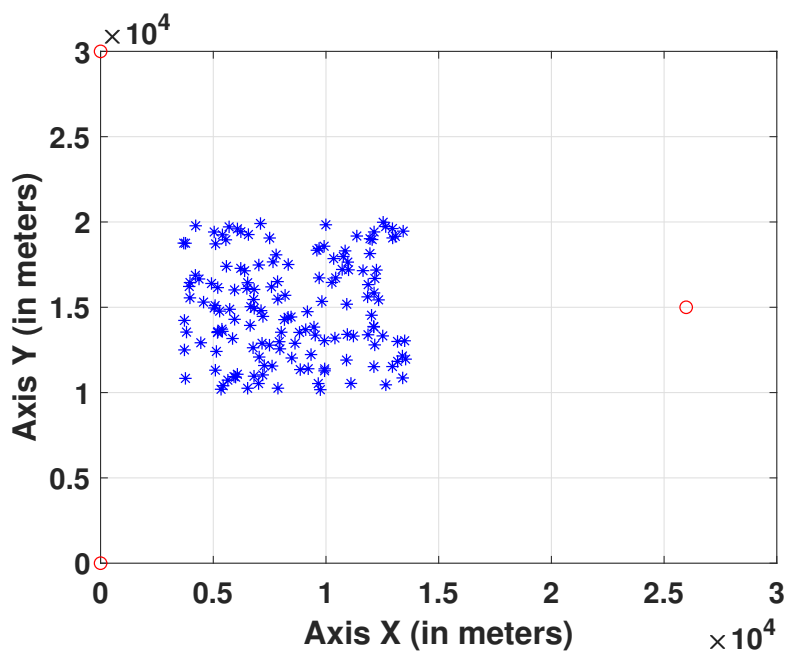

Fig. 6. An example of the extreme scenario. The blue points represent the users generated uniformly inside a $10 \mathrm{~km}$ square. The red points represent the eNodeBs.

2) Study-case 2: The goal is to show that the calibrated system-level simulator provides a highly accurate SINR analysis. For this purpose, the radio environment map (REM) of down-link SINR is presented in Fig. 7, where it is easy to visualize the high level of cell-interference between the 


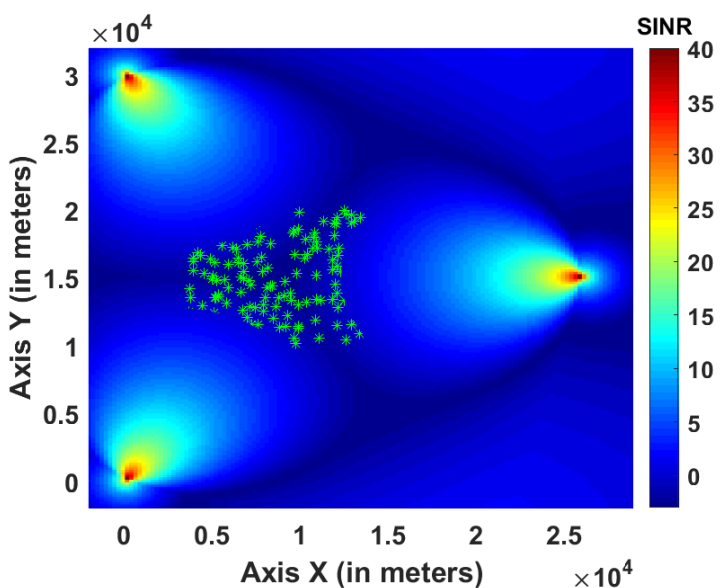

Fig. 7. High cell-interference setup used to evaluate frequency reuse techniques. The green asterisks represent the cellular users.

three sectors. The outcomes from the simulation were used to generate a probability density function (pdf) that models the throughput as a Gaussian random variable.

\section{NUMERICAL RESULTS}

The system-level simulator was used to obtain throughput $(T)$ in downlink for each user. This data was post-processed to obtain the mean using the next relationship:

$$
T_{\mathrm{avg}}=\frac{1}{N} \sum_{l=1}^{N} T_{l},
$$

where $T_{\text {avg }}$ is the aggregated average throughput (considering users attached to the 3 base stations), $T_{l}$ is the throughput of user $l$, and $N$ is the number of users considered in every configuration setup.

\section{A. Results of study-case 1}

From Fig. 8, it is possible to observe the average throughput of a typical cellular network for different number of simultaneous users. In this case, the best technique is the softfrequency reuse and the worst is the hard-frequency reuse. The same approach was considered to the extreme scenario. Fig. 9 shows different results for the same frequency reuse techniques presented before. In this last case, the hard frequency reuse outperforms all other options, while the worst solution is the enhanced fractional frequency reuse. This results indicate a special research attention in such extreme scenarios, mainly related to rural communication applied in energy systems distribution.

\section{B. Results of study-case 2}

The numerical results were obtained after the simulation of 100 different position snapshots (Monte-Carlo approach). We assume that the throughput $T$ is a Gaussian random variable with mean $\mu$ and variance $\sigma$ so that:

$$
p(x)=\mathcal{N}(x ; \mu, \sigma)=\frac{1}{\sqrt{2 \pi} \sigma} e^{-\frac{(x-\mu)^{2}}{2 \sigma^{2}}},
$$

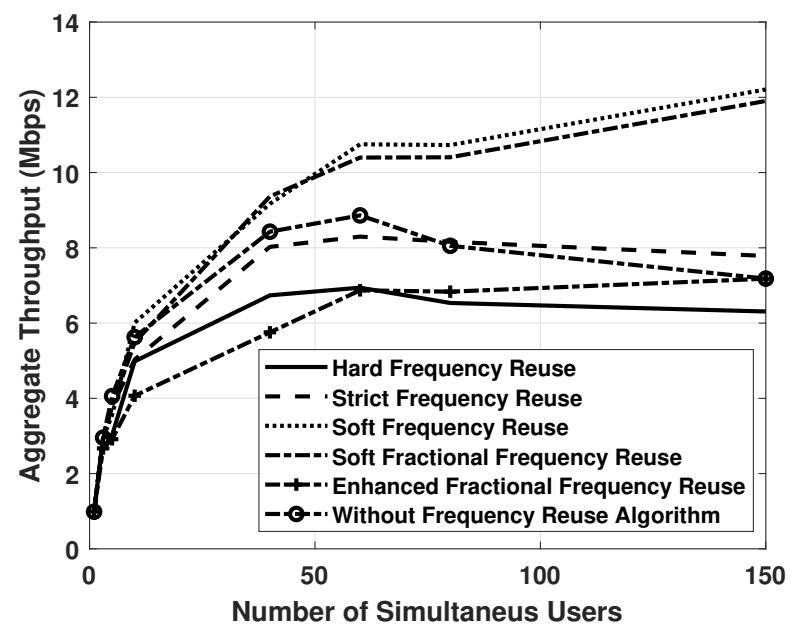

Fig. 8. Throughput comparison of frequency reuse techniques for a typical scenario of multi-carrier cellular network with different number of simultaneous users.

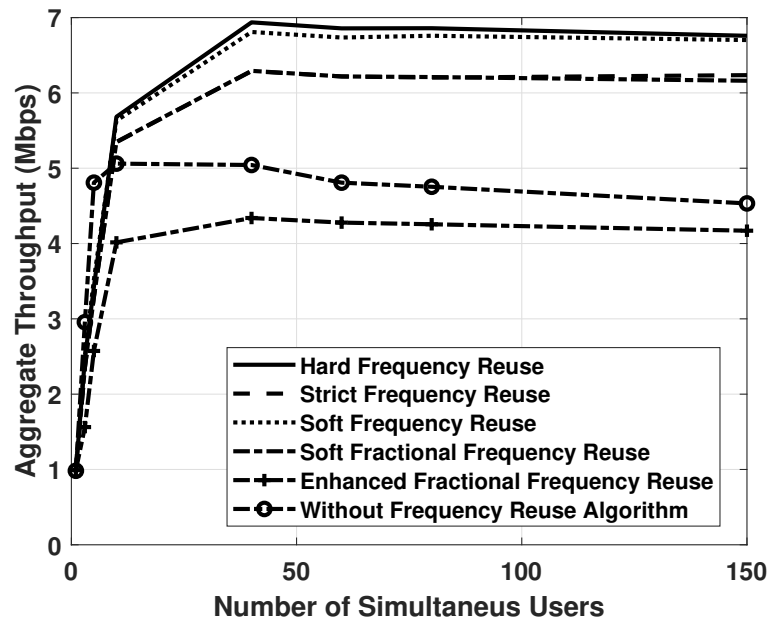

Fig. 9. Throughput comparison of frequency reuse techniques for a rare scenario of multi-carrier cellular network with different number of simultaneous users.

where $\mathcal{N}(x ; \mu, \sigma)$ is the notation of a Gaussian Function of random variable $x$ with mean $\mu$ and standard deviation $\sigma$. From the simulation outcomes, we compute the mean and variance values of $T$. We also plot the empirical histogram to validate the pdf model.

Fig. 10 shows the PDF model of one specific case where 150 users are deployed simultaneously in the $10 \mathrm{~km}$ square. In this case, the Gaussian function fits with a very good accuracy. This accuracy is further analyzed with the CDF in the Fig. 11. This result indicates a great potential to use stochastic tools to model the network performance. 


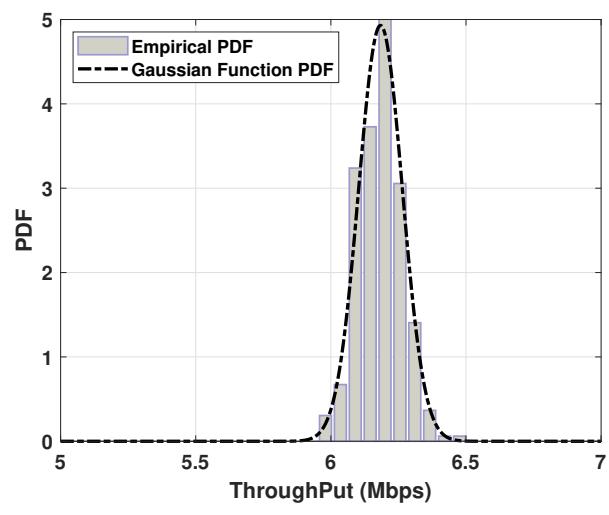

Fig. 10. Empirical and theory PDF for the random variable (average throughput) obtained by the system level simulator.

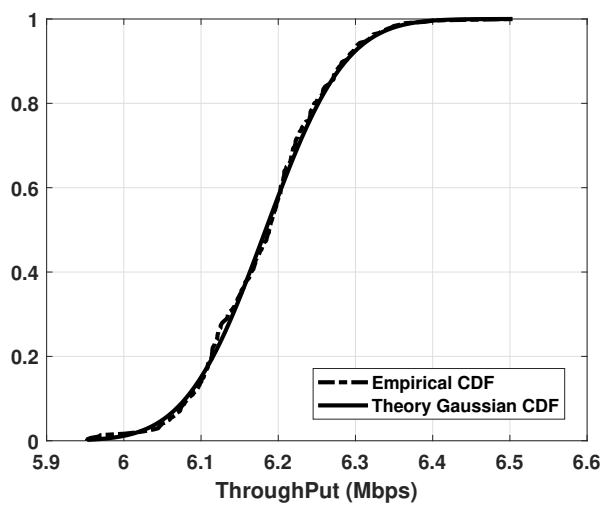

Fig. 11. Empirical and theory CDF for the random variable (average throughput) obtained by the system level simulator.

\section{CONCLUSIONS AND FUTURE WORKS}

Some multi-carrier cellular networks are being deployed in VHF to support rural area communications. Mostly of these networks are focused on providing connectivity to IoT applications in energy systems distribution or agribusiness market. This new cellular communication setups are creating new business models and simultaneously are generating extreme scenarios, which are the focus of this paper. Our initial research results showed that the same frequency reuse technique used in a typical cellular scenario obtains different results in rural communication extreme scenarios. In general, using a frequency reuse technique in a typical cellular scenario (with 150 users) obtained a throughput gain of $4 \mathrm{Mbps}$; in contrast, in the rare scenario the gain was $2 \mathrm{Mbps}$ approximately. We also obtained a theoretical PDF model of one random variable using system simulation results using a Gaussian function. This methodology and insight could be explored by future works using stochastic process theory. The aggregated throughput PDF analyzed in this work, could be extended to the study of marginal PDFs for each base station in this specific rare scenario. Other approach could use geometry stochastic theory to model these rare scenarios for cellular networks in VHF.

\section{ACKNOWLEDGEMENTS}

This work is partly funded by Academy of Finland via eeIoT project (ICT2023/n.319009).

\section{REFERENCES}

[1] V. Chandrasekhar, J. G. Andrews, and A. Gatherer, "Femtocell networks: a survey," IEEE Communications magazine, vol. 46, no. 9, 2008.

[2] S.-Y. Lien, S.-L. Shieh, Y. Huang, B. Su, Y.-L. Hsu, and H.-Y. Wei, "5G new radio: Waveform, frame structure, multiple access, and initial access," IEEE communications magazine, vol. 55, no. 6, pp. 64-71, 2017.

[3] A. Markhasin and V. Drozdova, "QoS-oriented multifunctional wireless mac for remote, rural, and difficult for access areas," in 2008 IEEE Region 8 International Conference on Computational Technologies in Electrical and Electronics Engineering, July 2008, pp. 389-394.

[4] R. Moreno, D. Carrillo, C. Lorena, and R. Coutinho, "IoT e conectividade para áreas remotas,” Portal: O Setor Elétrico, vol. 141, 2017.

[5] K. Mizutani, H. Kuriki, Y. Kodama, T. Matsumura, and H. Harada, "Performance evaluation of td-lte in vhf-band for large coverage public broadband communications system," in Wireless Personal Multimedia Communications (WPMC), 2017 20th International Symposium on. IEEE, 2017, pp. 80-86.

[6] M. Stoces, J. Vanek, J. Masner, and J. Pavlik, "Internet of things (IoT) in agriculture-selected aspects," Agris on-line Papers in Economics and Informatics, vol. 8, no. 1, p. 83, 2016.

[7] M. Lauridsen, I. Z. Kovács, P. Mogensen, M. Sorensen, and S. Holst, "Coverage and capacity analysis of LTE-M and NB-IoT in a rural area," in Vehicular Technology Conference (VTC-Fall), 2016 IEEE 84th. IEEE, 2016, pp. 1-5.

[8] J. Gozalvez, "New 3GPP standard for IoT [mobile radio]," IEEE Vehicular Technology Magazine, vol. 11, no. 1, pp. 14-20, 2016.

[9] C. L. Neto, E. J. Bonon, and F. L. Figueiredo, "Brazilian telecommunications regulatory framework and the impacts on the development of broadband radio access systems," in Long Term Evolution - 4G and Beyond, A. Paradisi, M. D. Yacoub, F. L. Figueiredo, and T. Tronco, Eds. Springer, 2015, ch. 2, pp. 27-44.

[10] Y. Shifeng, F. Chungui, H. Yuanyuan, and Z. Shiping, "Application of IoT in agriculture," Journal of Agricultural Mechanization Research, vol. 7, pp. 190-193, 2011.

[11] P. P. Ray, "Internet of things for smart agriculture: Technologies, practices and future direction," Journal of Ambient Intelligence and Smart Environments, vol. 9, no. 4, pp. 395-420, 2017.

[12] S. Gansemer, J. Sell, U. Grossmann, E. Eren, B. Horster, T. HorsterMöller, and C. Rusch, M2M in Agriculture-Business Models and Security Issues. The River Publishers Series in Automation, Control and Robotics. River Publishers, 2015.

[13] The ns-3 network simulator, http://www.nsnam.org/. Last time accessed: October 2018.

[14] H. Holma and A. Toskala, LTE for UMTS: Evolution to LTE-advanced. John Wiley \& Sons, 2011.

[15] A. Larmo, M. Lindström, M. Meyer, G. Pelletier, J. Torsner, and H. Wiemann, "The LTE link-layer design," IEEE Communications Magazine, vol. 47, no. 4, pp. 52-59, April 2009.

[16] F. Thiesse, C. Floerkemeier, M. Harrison, F. Michahelles, and C. Roduner, "Technology, standards, and real-world deployments of the EPC network," IEEE Internet Computing, vol. 13, no. 2, pp. 36-43, 2009.

[17] D. Astély, E. Dahlman, A. Furuskär, Y. Jading, M. Lindström, and S. Parkvall, "LTE: the evolution of mobile broadband," IEEE Communications magazine, vol. 47, no. 4, 2009.

[18] X. Guo, R. Rouil, C. Soin, S. Parekh, B. Sikdar, and S. Kalyanaraman, "WiMAX system design and evaluation methodology using the ns-2 simulator," in Proceedings of the First International Conference on COMmunication Systems And NETworks, ser. COMSNETS'09. Piscataway, NJ, USA: IEEE Press, 2009, pp. 643-652.

[19] S. Yi, S. Chun, Y. Lee, S. Park, and S. Jung, Overview of LTE and LTE-Advanced New Features. Wiley, 2013.

[20] A. S. Hamza, S. S. Khalifa, H. S. Hamza, and K. Elsayed, "A Survey on Inter-Cell Interference Coordination Techniques in OFDMA-Based Cellular Networks," IEEE Communications Surveys Tutorials, vol. 15, no. 4, pp. 1642-1670, Fourth 2013. 